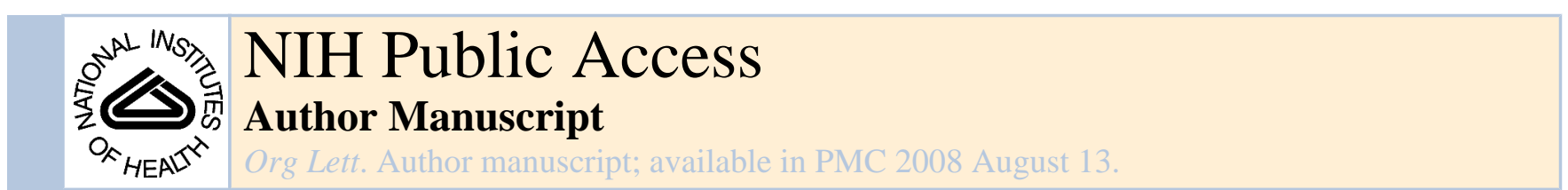

Published in final edited form as:

Org Lett. 2007 April 12; 9(8): 1597-1600.

\title{
Aminomethylations via Cross-Coupling of Potassium Organotrifluoroborates with Aryl Bromides
}

\author{
Gary A. Molander and Deidre L. Sandrock \\ Roy and Diana A. Vagelos Laboratories, Department of Chemistry, University of Pennsylvania, \\ Philadelphia PA, 19104-6323, gmolandr@sas.upenn.edu
}

\section{Abstract}

The Suzuki-Miyaura cross-coupling reaction of $N, N$-dialkylaminomethyltrifluoroborates with aryl halides allows the construction of an aminomethyl aryl linkage through a disconnection based on dissonant reactivity patterns. A variety of these aminomethyltrifluoroborate substrates were prepared in good to excellent yields and then shown to cross-couple with equal facility to both electron-rich and electron-poor aryl halides as well as to a variety of heteroaromatic bromides.

\begin{abstract}
Alkaloid natural products and nitrogen-containing pharmaceuticals are highly prominent synthetic targets. ${ }^{1}$ This is a direct result not only of the structural complexity that many of these molecules possess, but also of their marked biological activity. The properties associated with the nitrogen atom itself, however, often make alkaloid synthesis a more painstaking endeavor than the synthesis of polyketide- or terpene-derived structures. Thus new, facile methods of nitrogen incorporation are in high demand.
\end{abstract}

Many bioactive molecules possess an aminomethyl alkenyl- or aryl linkage (Figure 1). There is ample precedent for the installation of this aminomethyl moiety by nucleophilic displacement ${ }^{2}$ alkylation of iminium ions, or reductive amination. ${ }^{3}$ These more classical strategies possess consonant reactivity patterns in line with the polarities of the functional groups involved (eqs 1-2).

Although these tactics have been employed extensively and effectively, a new strategy employing a fundamentally different aminomethyl disconnection would provide a complementary (dissonant) C-C bond construction (eq 3). Consideration of dissonant disconnects logically leads to a class of carbanions that are generally difficult to access, somewhat unstable, and consequently only employed to a limited extent in this context.

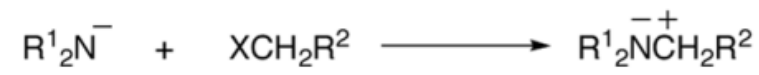

$\mathrm{R}_{2}{ }_{2}^{\stackrel{+}{\mathrm{N}}}=\mathrm{CH}_{2}+{ }^{-} \mathrm{CH}_{2} \mathrm{R}^{2} \longrightarrow \mathrm{R}_{2}^{1}{ }_{2} \stackrel{+}{\mathrm{N}^{2}}{ }_{2}-\overline{\mathrm{C}}_{2} \mathrm{R}^{2}$ 


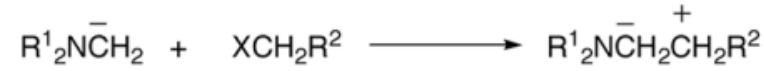

Fortunately, cross-coupling reactions and the organometallics used therein possess the potential to circumvent these limitations. To date, however, a single cross-coupling has been reported that takes advantage of this type of transformation (eq 4).
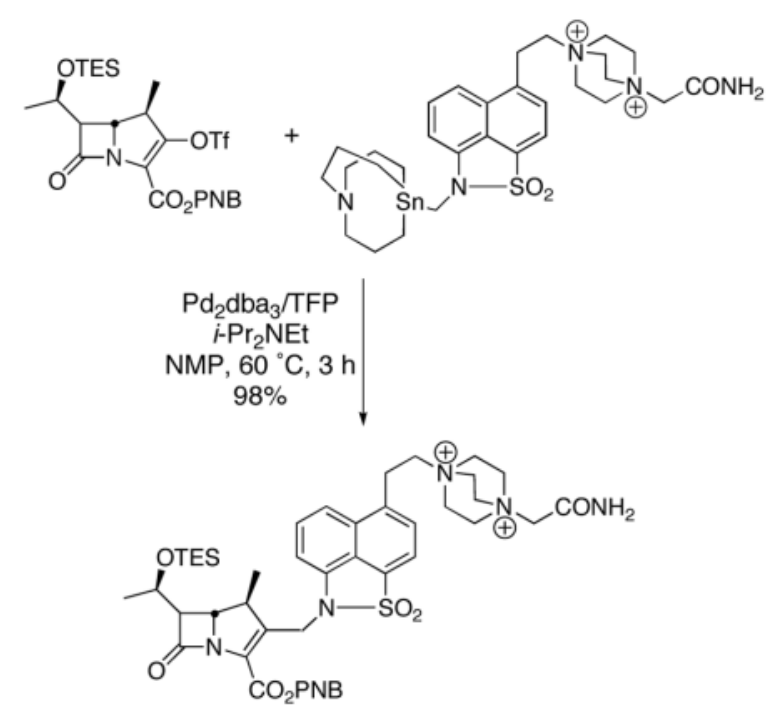

This procedure partnered a highly specialized aminomethylstannane and an enol triflate. ${ }^{4}$ Because of the complexity of this reagent, the inherent toxicity associated with organotin reagents, as well as difficulties associated with purification of the resulting cross-coupled product, the Stille reaction is a less than ideal aminomethylating platform. The possibility of applying nontoxic, air- and moisture stable potassium $N, N$ -

dialkylaminomethyltrifluoroborates makes Suzuki-Miyaura cross-couplings with organic halides an attractive alternative. ${ }^{5}$ Herein, preliminary studies toward that end are disclosed.

Initial analysis focused on the use of potassium $N$-(trifluoroboratomethyl)piperidine, which is easily prepared according to a previously published procedure. ${ }^{6}$ Optimization was conducted utilizing 4-bromobenzonitrile 1a) as the electrophile. After investigating several catalyst and ligand systems, the combination of $3 \mathrm{~mol} \%$ of $\mathrm{Pd}(\mathrm{OAc})_{2}$ and $6 \mathrm{~mol} \%$ of 2-

dicyclohexylphosphino-2', $4^{\prime}, 6^{\prime}$-triisopropylbiphenyl (XPhos) ${ }^{7}$ was determined to be the most effective catalyst system. $\mathrm{Cs}_{2} \mathrm{CO}_{3}$ (3 equiv) and a 10:1 THF/ $\mathrm{H}_{2} \mathrm{O}$ mixture proved to be a satisfactory base and solvent, respectively. 2 a could be produced in $83 \%$ yield under these conditions (Table 1 , entry 1 ).

To investigate the method further, both electron-poor (Table 1, entries 1-5) and electron-rich (Table 1, entries 6-10) aryl halides were examined. Both were found to cross-couple with equal facility, providing the aminomethylated products in good to excellent yields. A variety of functional groups including nitriles, esters, ketones, aldehydes, amides, and amines were successfully incorporated within the electrophiles. The effects of steric hindrance were probed using mesityl bromide as the electrophile. Thus, despite the presence of two ortho substituents, 1h was found to couple effectively, yielding $\mathbf{2 h}$ in $69 \%$ isolated yield (Table 1, entry 8). 
To improve the yields in some of the transformations, an alternative solvent system consisting of cyclopentyl methyl ether (CPME) and water was investigated. In all cases, use of the CPME/ $\mathrm{H}_{2} \mathrm{O}$ solvent system (10:1) increased the yield and decreased the reaction time, presumably because it allowed a higher reaction temperature (Table 1, entries 3-5, 8, and 10). This solvent system offers greatly improved yields for the coupling of 2-bromomesitylene and 4-bromo$N, N$-dimethylaniline (1j) to yield $\mathbf{2 h}(77 \%)$ and $\mathbf{2 j}(82 \%)$, respectively.

To expand the array of electrophiles that can be utilized, we coupled the potassium $\mathrm{N}$ (trifluoroboratomethyl)-piperidine with a variety of heteroaromatic bromides (Table 2). We found that the highest yields of these substrates could be obtained by using the original THF/ $\mathrm{H}_{2} \mathrm{O}$ solvent system conditions. An assortment of these substrates were examined including furan-, thiophene-, pyrimidine-, isoquinoline-, and pyridine derivatives. The resulting aminomethylated heteroaromatics were obtained in modest to good yields. 5-Bromo-2furaldehyde (3a) and 5-bromopyrimidine (3d) coupled with the greatest facility to afford coupled products $\mathbf{4 a}$ and $\mathbf{4 d}$, respectively.

To demonstrate the scope of this method further, we prepared a variety of potassium $N, N$ dialkylaminomethyl-trifluoroborates (Table 3 ) to use as coupling substrates. We have previously disclosed a method of aminomethyltrifluoroborate preparation that necessitated the use of superstoichiometric quantities of the amine, ${ }^{6}$ which, though functional, cannot be applied on a synthetically useful scale with valuable amines. Table 3 (entries 3-5) illustrates stoichiometric reaction conditions, which yield tertiary amine products in good yields.

Both cyclic and acyclic aminomethyltrifluoroborates were found to be effective coupling partners using the conditions presented here (Table 4$)$. Of special interest are the successes in coupling the benzyl-protected $N$-methylaminomethyl- $(\mathbf{5 c})$ and the protected piperazinomethyl- (5d) trifluoroborate derivatives to yield the respective products, both of which offer the potential for further functionalization of the amines following deprotection.

Though the proline methyl ester- and diallylamine nucleophiles were acceptable substrates for the preparation of their trifluoroborate derivatives (Table 3, entries 5 and 6) initial studies have shown that they were ineffective substrates with the optimized coupling conditions in both $\mathrm{THF} / \mathrm{H}_{2} \mathrm{O}$ and $\mathrm{CPME} / \mathrm{H}_{2} \mathrm{O}$ solvent systems.

In conclusion, we have synthesized a variety of potassium dialkylaminomethyltrifluoroborates and demonstrated their suitability as coupling partners in Suzuki-Miyaura cross-coupling reactions with both electron-rich and electron-poor aryl bromides. The ease with which these potassium organotrifluoroborates can be prepared through a synthetically useful one-step process from bromomethyltrifluoroborate ${ }^{6}$ provides a new means for accessing structurally significant nitrogen-containing molecules. Efforts toward the further elaboration of this method, as well as applications to complex molecule synthesis, are currently underway.

\section{Supplementary Material}

Refer to Web version on PubMed Central for supplementary material.

\section{Acknowledgements}

The authors thank NIH (GM 35249), Amgen, Johnson \& Johnson, and Merck Research Laboratories for their generous support of our program. Johnson Matthey is acknowledged for their donation of palladium catalysts, Zeon for their donation of CPME, and Professor Stephen L. Buchwald (MIT) for a donation of phosphine ligands. Dr. Rakesh Kohli (University of Pennsylvania) is acknowledged for obtaining HRMS data. 


\section{References}

1. Kibayashi C. Chem Pharm Bull 2005;53:1375. [PubMed: 16272717]

2. Zhao P, Yin YW. J Heterocycl Chem 2004;41:157.

3. (a) Abdel-Magid AF, Carson KG, Harris BD, Maryanoff CA, Shah RD. J Org Chem 1996;61:3849. [PubMed: 11667239] (b) Abdel-Magid AF, Mehrman SJ. Org Process Res Dev 2006;10:971.

4. Jensen MS, Yang C, Hsiao Y, Rivera N, Wells KM, Chung JYL, Yasuda N, Hughes DL, Reider PJ. Org Lett 2000;2:1081. [PubMed: 10804559]

5. Suzuki, A.; Brown, HC. Organic Syntheses via Boranes: Volume 3 Suzuki Coupling. Aldrich Chemical Co.; Milwaukee: 2003. (b) Miyaura N. Top Curr Chem 2002;219:11. (c) Miyaura N, Suzuki A. Chem Rev 1995;95:2457. (c) Molander GA, Figueroa R. Aldrichimica Acta 2005;38:49. (d) Molander GA, Ellis N. Acc Chem Res, ASAP. (e) Stefani HA, Cella R, Vieira AS. Tetrahedron. in press

6. Molander GA. Ham, J Org Lett 2006;8:2031.

7. (a) Milne JE, Buchwald SL. J Am Chem Soc 2004;126:13028. [PubMed: 15469301] (b) Barder TE, Walker SD, Martinelli JR, Buchwald SL. J Am Chem Soc 2005;127:4685. [PubMed: 15796535] 
<smiles>[R]C1Cc2nc3c(nc2[CH-]CN1)C[C@@H]([R])NC3</smiles>

barrenazines<smiles>C[14CH2]c1cncc2[nH]ccc12</smiles>

Amgen VEGF inhibitor<smiles>[R6]Oc1cc2c(cc1O)C1CC([R6])CC=C1CN2C</smiles>

lycoranes<smiles>[R]O[C@H]1CCN2C[CH+]C=C([R])C12</smiles>

pyrrolizidines<smiles>COc1cc2c(cc1OC)C(=O)C(CC1CCN([CH+]c3ccccc3)CC1)C2</smiles>

Figure 1.

Dissonant disconnects for the construction of nitrogen-containing compounds. 
Table 1

Cross-Coupling of Potassium $N$-(Trifluoroboratomethyl)piperidine with Various Aryl Halides ${ }^{a}$

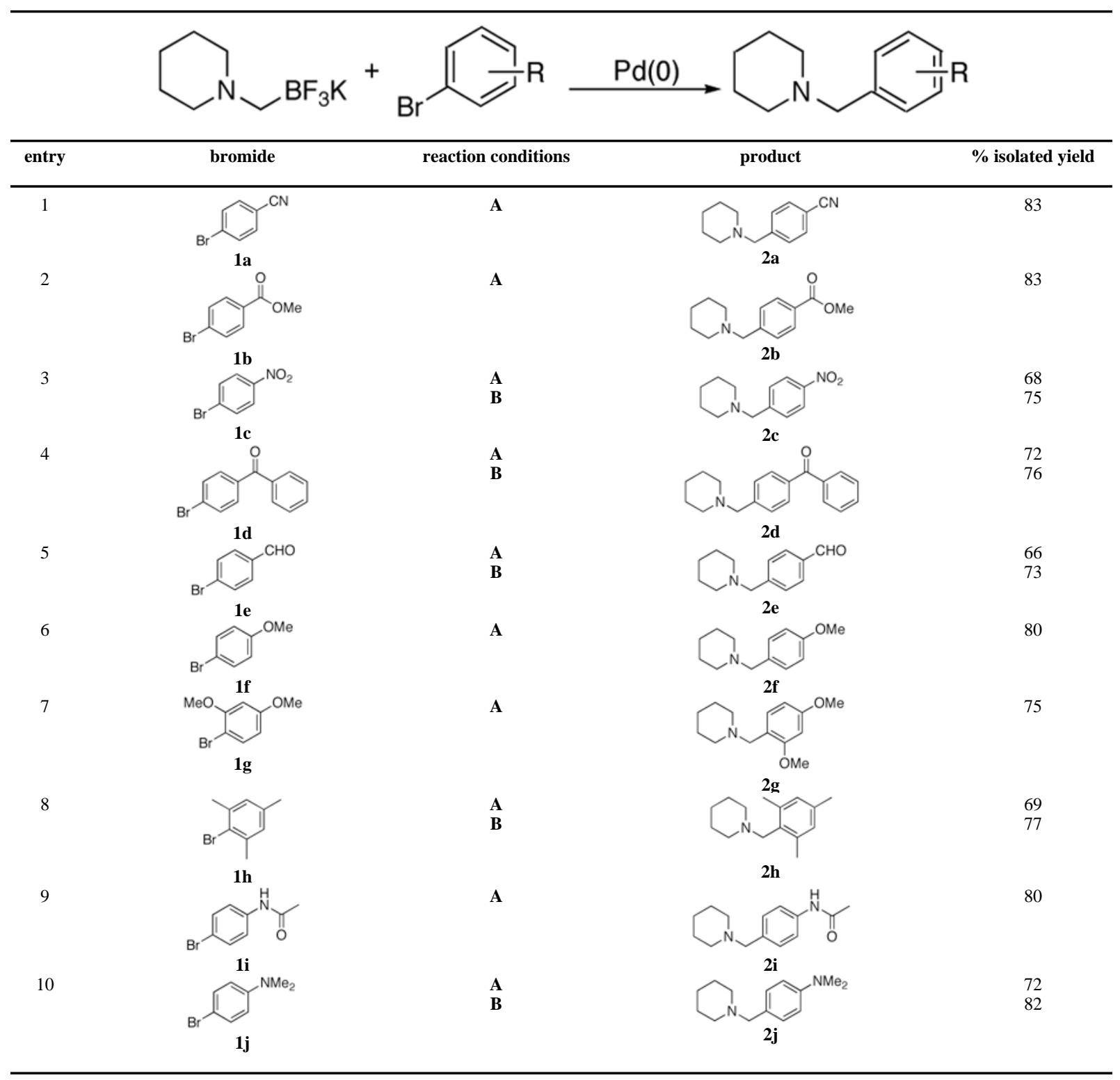

${ }^{a}$ Conditions: All used $\mathrm{Pd}(\mathrm{OAc})_{2}(3 \mathrm{~mol} \%)$, XPhos (6 mol \%), $\mathrm{Cs}_{2} \mathrm{CO}_{3}$ (3.0 equiv), and $0.25 \mathrm{M}$ solvent system; $\mathbf{A}: 10: 1 \mathrm{THF} / \mathrm{H}_{2} \mathrm{O}, 80^{\circ} \mathrm{C}, 22-24 \mathrm{~h} ; \mathbf{B}$ : 10:1 CPME/ $/ \mathrm{H}_{2} \mathrm{O}, 95^{\circ} \mathrm{C}, 12-18 \mathrm{~h}$. 
Table 2

Cross-Coupling of Potassium $N$-(Trifluoroboratomethyl)piperidine with Various Heteroaromatic Bromides ${ }^{a}$

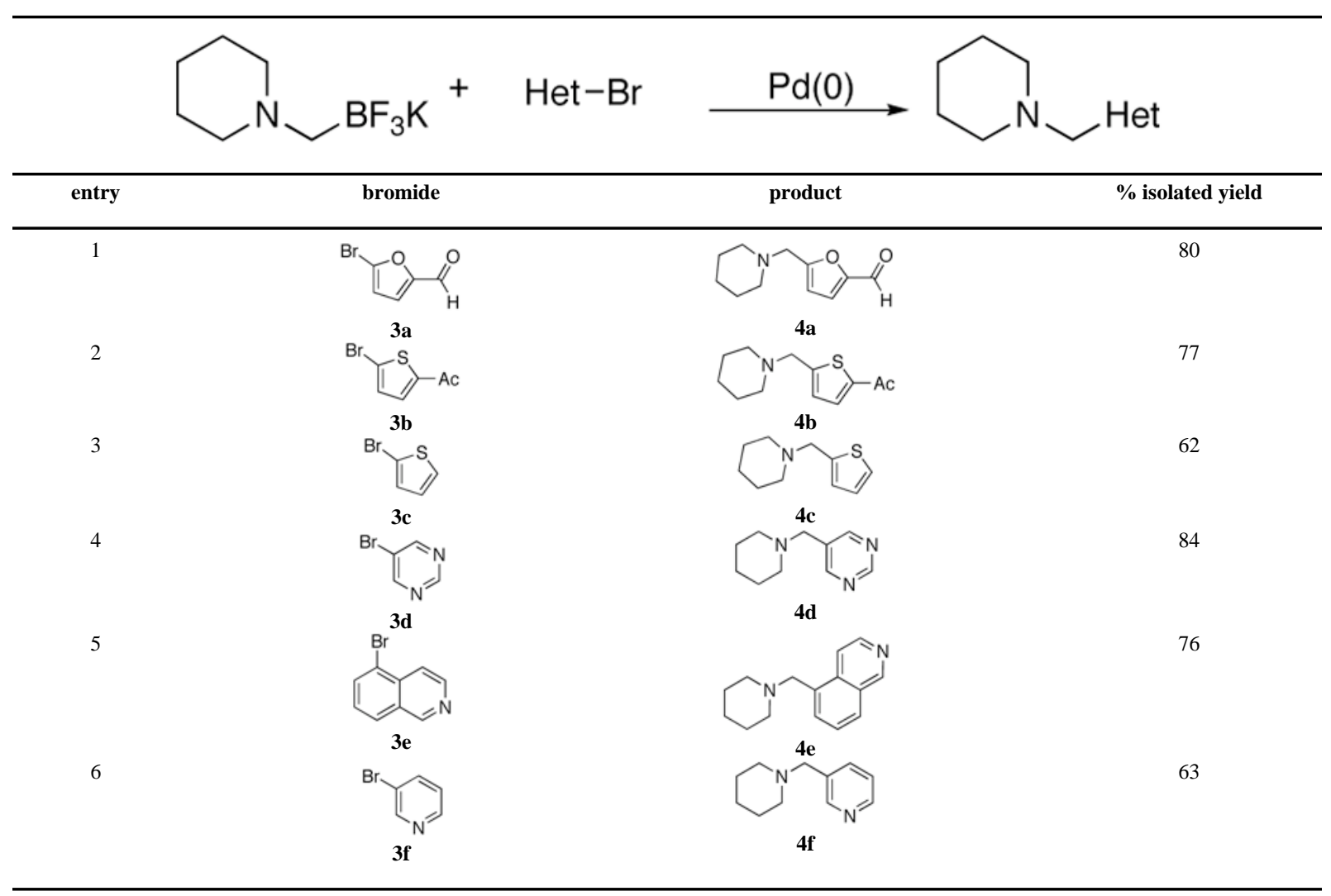

${ }^{a}$ Conditions: All used $\mathrm{Pd}(\mathrm{OAc}) 2$ (3 mol \%), XPhos (6 mol \%), $\mathrm{Cs}_{2} \mathrm{CO}_{3}$ (3.0 equiv), and 10:1 THF/H2O $(0.25 \mathrm{M}), 80^{\circ} \mathrm{C}, 18-24 \mathrm{~h}$. 


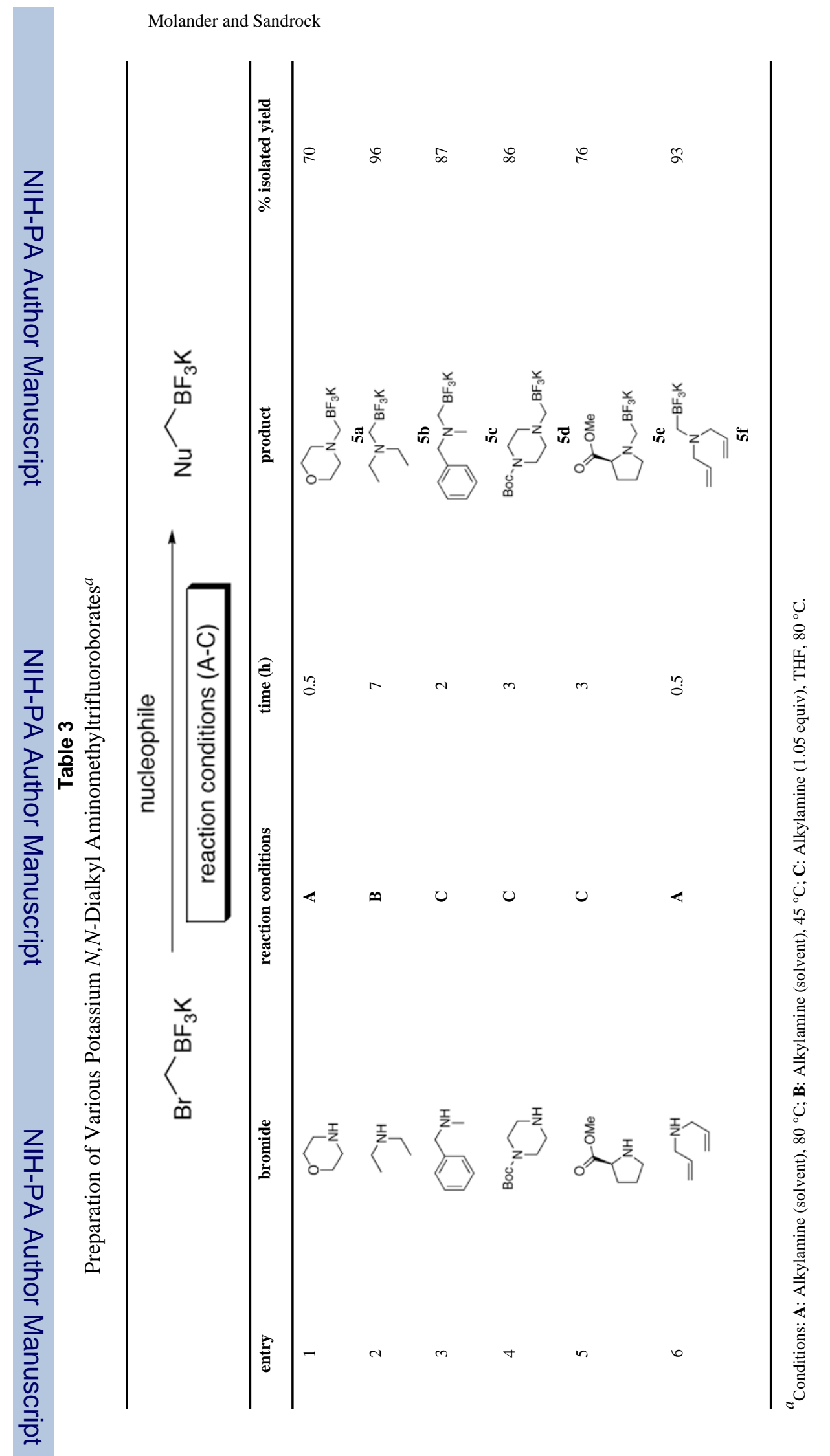

Org Lett. Author manuscript; available in PMC 2008 August 13. 
Table 4

Cross-Coupling of $N, N$-Dialkyl Aminomethyl-trifluoroborates with 4-Bromoanisole ${ }^{a}$

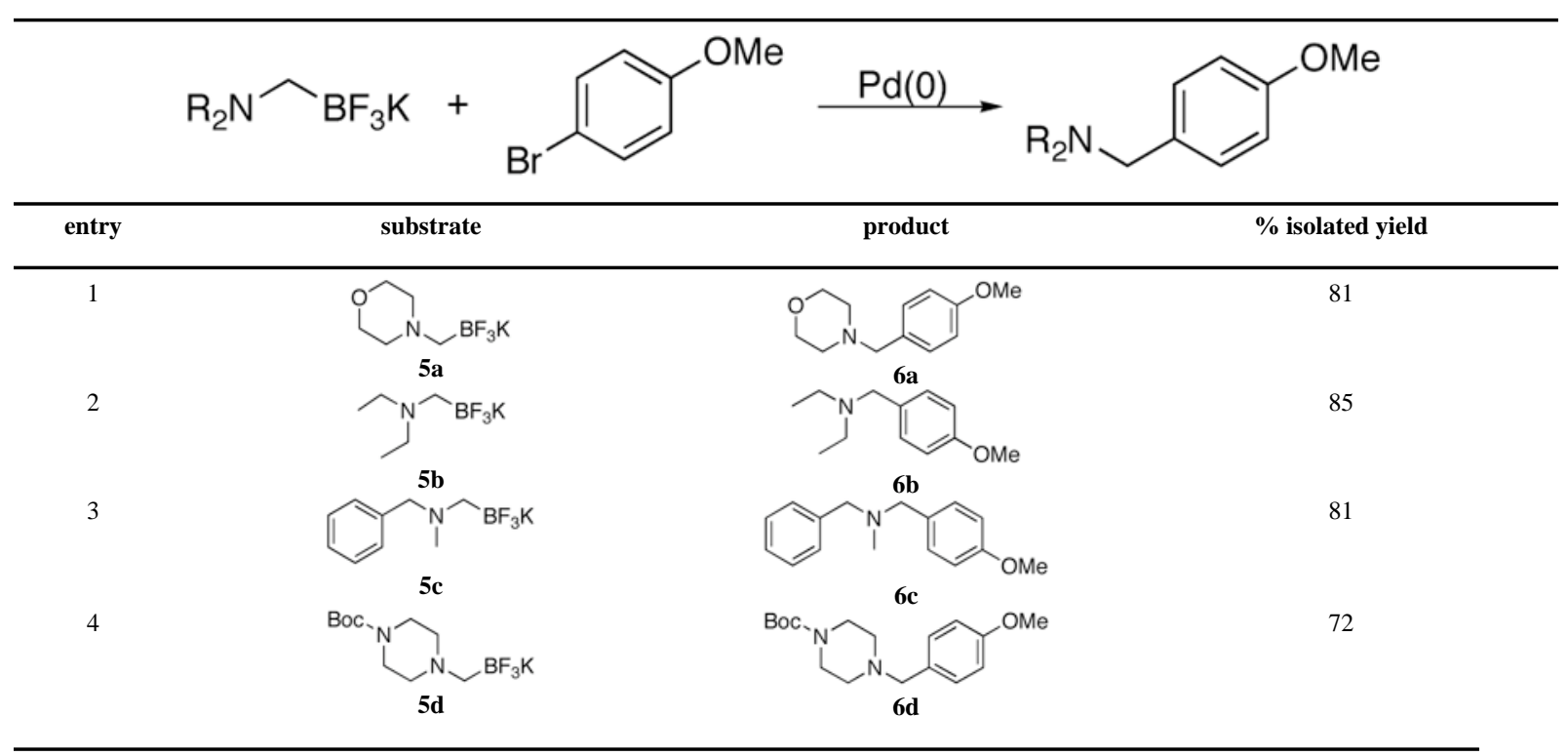

${ }^{a}$ Conditions: All used $\mathrm{Pd}(\mathrm{OAc}) 2$ (3 mol \%), XPhos (6 mol \%), $\mathrm{Cs}_{2} \mathrm{CO}_{3}$ (3.0 equiv), and 10:1 THF/ $\mathrm{H}_{2} \mathrm{O}(0.25 \mathrm{M}), 80^{\circ} \mathrm{C}, 18-24 \mathrm{~h}$. 